\title{
An Easy and Efficient Method to Produce $\gamma$-Amino Alcohols by Reduction of $\beta$-Enamino Ketones
}

\author{
Maria Inês N. C. Harris and Antonio C. H. Braga* \\ Instituto de Química, Universidade Estadual de Campinas, CP 6154, 13083-970 Campinas -SP, Brazil
}

\begin{abstract}
A redução de $\beta$-enamino cetonas 2 com $\mathrm{NaBH}_{4}$ em ácido acético glacial produziu $\gamma$-amino álcoois $1 \mathrm{em} 70 \%$ a $98 \%$ de rendimento, com excessos diastereoméricos, preferencialmente o produto syn, de $44 \%$ a $90 \%$. A estereoquímica desses compostos foi confirmada pela análise de seus derivados tetraidro-1,3-oxazinas 3 .
\end{abstract}

Reduction of $\beta$-enamino ketones $\mathbf{2}$ with $\mathrm{NaBH}_{4}$ in glacial acetic acid gave $\gamma$-amino alcohols $\mathbf{1}$ in $70 \%$ to $98 \%$ yield with diastereomeric excesses, preferentially the syn product, from $44 \%$ to $90 \%$. The stereochemistry of these compounds was confirmed by analysis of their tetrahydro-1,3-oxazine derivatives 3 .

Keywords: amino alcohols, enamino ketones, oxazines, stereoselective reduction

\section{Introduction}

The synthesis of $\gamma$-amino alcohols $\mathbf{1}$ is of great interest due to the pharmacology of these compounds and their derivatives. This functionality is found in several antibiotics and other biologically active natural products. ${ }^{1}$ Several synthetic methods have been described for the synthesis of $\gamma$-amino alcohols $\mathbf{1}$ from diols, ${ }^{2}$ hydroxazols, ${ }^{3}$ lactams ${ }^{4}$ and lactones, ${ }^{5}$ but the more important methods are those where one can obtain $\gamma$-amino alcohols $\mathbf{1}$ by reduction of 1,3-difunctionalized unsaturated compounds containing nitrogen and oxygen, such as $\beta$-hydroxy oximes, ${ }^{6} \beta$-enamino ketones $2,{ }^{7-11}$ and, more frequently, by the reduction of $\beta$-amino ketones. ${ }^{1,12-15}$

$\gamma$-Amino alcohols $\mathbf{1}$, mainly syn, can be synthesized by reduction of $\beta$-enamino ketones 2 with $\mathrm{Na}$ in $\mathrm{Pr}$ i $\mathrm{OH} /$ tetrahydrofuran or with $\mathrm{CeCl}_{3} / \mathrm{LiBH}_{4}$ /tetrahydrofuran. ${ }^{9} \mathrm{On}$ the other hand, the combination of $\mathrm{NaBH}_{4}$ in a carboxylic acid media has yielded an efficient reducing reagent. ${ }^{16}$

We wish to report herein a simple and efficient method to produce $\gamma$-amino alcohols $\mathbf{1}$ through the reduction of $\beta$-enamino ketones 2 with $\mathrm{NaBH}_{4}$ in glacial acetic acid, which has been sucessfully used in our laboratory. ${ }^{17}$

\section{Results and Discussion}

Difficulties in reduction of $\beta$-enamino ketones 2 have

*e-mail: herrera@iqm.unicamp.br been reported. ${ }^{10}$ The use of $\mathrm{NaBH}_{4}$ in a carboxylic acid medium is well known, ${ }^{16}$ but its use in the reduction of $\beta$-enamino ketones $\mathbf{2}$ has not been explored. Our results show that the reaction of $\beta$-enamino ketones 2 with $\mathrm{NaBH}_{4}$ in glacial acetic acid ( 3 hours at room temperature, Scheme 1 ), produces a mixture of syn/anti $\gamma$-amino alcohols $\mathbf{1}$, the syn isomer being the major product (Table 1). ${ }^{18}$

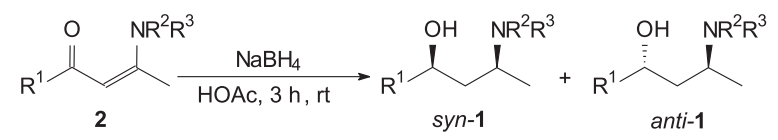

Scheme 1.

Table 1. Diastereomeric ratios of $\gamma$-amino alcohols 1 in the reduction of $\beta$-enamino ketones 2

\begin{tabular}{llllcc}
\hline $\mathbf{2}$ & $\mathrm{R}^{1}$ & $\mathrm{R}^{3}$ & $\mathrm{R}^{2}$ & $\mathbf{\%} \mathbf{1}^{\mathrm{c}}$ & syn/anti $^{\mathrm{a}}$ \\
\hline $\mathbf{a}$ & $\mathrm{Me}$ & $\mathrm{H}$ & $\mathrm{Ph}$ & 98 & $87 / 13$ \\
$\mathbf{b}$ & $\mathrm{Me}$ & $\mathrm{H}$ & $\mathrm{Bn}$ & 90 & $80 / 20$ \\
c & $\mathrm{Me}$ & $\mathrm{H}$ & ${ }^{\mathrm{Pr}}$ & 98 & $72 / 28$ \\
d & $\mathrm{Ph}$ & $\mathrm{H}$ & ${ }^{\mathrm{Pr}}$ & 85 & $90 / 10$ \\
f & $\mathrm{T}$ & $\mathrm{H}$ & $\mathrm{Bn}$ & 70 & $>95 / 5^{\mathrm{b}}$ \\
e & $\mathrm{Me}$ & $-\left(\mathrm{CH}_{2}\right)_{4}^{-}$ & 93 & $75 / 25$ \\
\hline
\end{tabular}

a Diastereomeric ratio determined in the mixtures of the tetrahydro1,3-oxazine derivatives 3 using ${ }^{13} \mathrm{C}$ NMR $(75,1 \mathrm{MHz})$, and confirmed by CG/MS; ${ }^{\text {b }}$ 1f was not isolated; it was transformed immediately into $\mathbf{3 f}$; ${ }^{c}$ isolated yield.

When the reaction is carried out without temperature control, the reaction produces the $\alpha, \beta$-unsaturated ketone 6 while at $0{ }^{\circ} \mathrm{C}$ (hexane/HOAc, $\mathrm{CH}_{2} \mathrm{Cl}_{2} / \mathrm{HOAc}$ or HOAc as 
solvent) the product is a mixture of reactant $2, \gamma$-amino alcohol 1 and the corresponding Mannich base 5. Another important observation is that, by this methodology, it is impossible to reduce 3-(N-benzylamino)-2-cyclohexen-1one.

A mechanism is suggested where chelated intermediate $\mathbf{4}$ is reduced to produce $\beta$-amino ketone $\mathbf{5}$ and $\gamma$-amino alcohol 1 (Scheme 2).

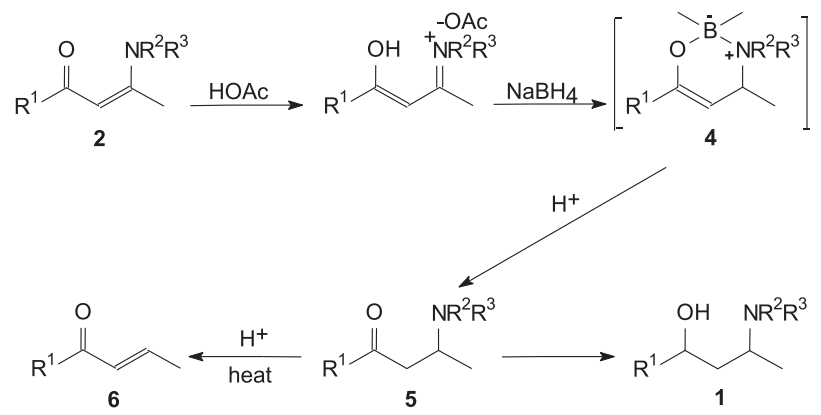

Scheme 2.

Quantitative conversion of $\gamma$-amino alcohols 1 to the corresponding tetrahydro-1,3-oxazine derivatives $\mathbf{3}$ (formol in diethyl ether, Scheme 3), ${ }^{19}$ allow us to assign the syn stereochemistry to the major $\gamma$-amino alcohol $\mathbf{1}$ after inspection of their ${ }^{1} \mathrm{H}$ and ${ }^{13} \mathrm{C}$ NMR spectra. Using the $s y n-\mathbf{3 a}$ compound as an example (Scheme 4), we can see the hydrogen atoms $\mathrm{H}_{4}$ and $\mathrm{H}_{6}$ as a double quartet of doublets at $3.27 \mathrm{ppm}$ $(J 3.3,6.3,11.1 \mathrm{~Hz})$ and $3.68 \mathrm{ppm}(J 2.7,6.3,12.3 \mathrm{~Hz})$ respectively. These hydrogen atoms and $\mathrm{H}_{5 \mathrm{e}}$ are in a axialequatorial situation $\left(\theta \cong 60^{\circ}\right)$, with coupling constants $2.7 \mathrm{~Hz}$ $\left(J_{\mathrm{H} 4-\mathrm{H} 5 \mathrm{e}}\right)$ and $3.3 \mathrm{~Hz}\left(J_{\mathrm{H} 6-\mathrm{H} 5 \mathrm{e}}\right)$. The ${ }^{1} \mathrm{H}$ NMR spectrum shows the axial-axial $\left(\theta \cong 180^{\circ}\right)$ relation between $\mathrm{H}_{5 \mathrm{a}}$ and hydrogen atoms $\mathrm{H}_{6}$ and $\mathrm{H}_{4}\left(J_{\mathrm{H} 4-\mathrm{H} 5 \mathrm{a}} 11.1 \mathrm{~Hz} ; J_{\mathrm{H} 6-\mathrm{H} 5 \mathrm{a}} 12.5 \mathrm{~Hz}\right)$. Furthermore we can see $\mathrm{H}_{5 \mathrm{e}}$ and $\mathrm{H}_{5 \mathrm{a}}$ as a double triplet at $1.57 \mathrm{ppm}(J$ 2.7, $13.5 \mathrm{~Hz})$ and $1.42 \mathrm{ppm}(J 11.1,13.0 \mathrm{~Hz})$, respectively. The analysis of the ${ }^{13} \mathrm{C}$ NMR spectrum (Scheme 5) allows the assignement of the secondary carbons at $40.03 \mathrm{ppm}$ and 85.76 $\operatorname{ppm}\left(\mathrm{C}_{5}\right.$ and $\mathrm{C}_{2}$ respectively), and the tertiary carbons at 53.90 ppm and 73.43 ppm $\left(\mathrm{C}_{4}\right.$ and $\mathrm{C}_{6}$ respectively). The chemical shifts of the methyl groups were assigned mainly based on the protective anisotropic effect of the phenyl group at $\mathrm{C}_{4}$. The chemical shift of the carbon $\mathrm{C}_{6}$ in the syn-3a isomer and in the anti-3a isomer are 73.43 and 68.14 ppm respectively. This upfield shift (ca. $5 \mathrm{ppm}$ ) is compatible with a $\gamma$-gauche exocyclic interaction, showing the axial methyl group at $\mathrm{C}_{4}$.

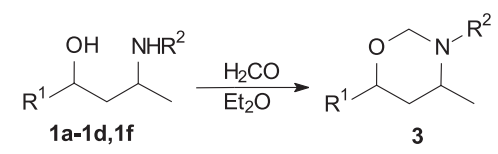

Scheme 3.

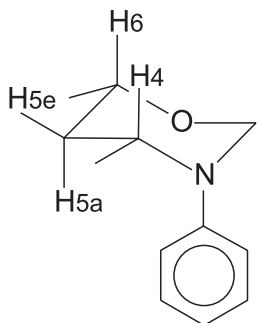

syn-3a

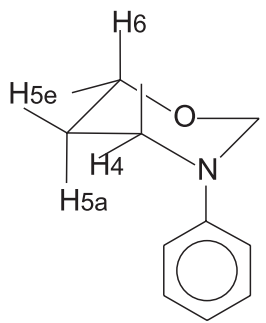

anti-3a
Scheme 4.<smiles>CC1CC(C)N(c2ccccc2)CO1</smiles>

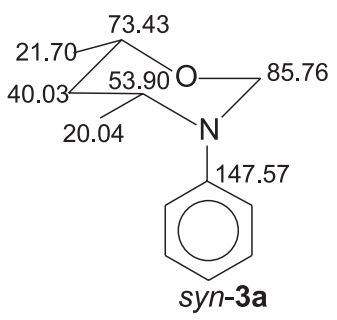

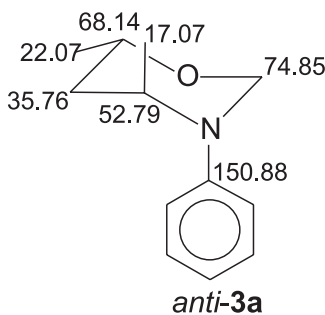

Scheme 5.

In conclusion, the reduction of $\beta$-enamino ketones $\mathbf{2}$ with $\mathrm{NaBH}_{4}$ in acetic acid is a very simple and fast method to obtain $\gamma$-amino alcohols $\mathbf{1}$ (with preferential syn configuration) in good chemical yields.

\section{Experimental}

\section{General}

${ }^{1} \mathrm{H}$ NMR and ${ }^{13} \mathrm{C}$ NMR spectra were recorded on a GEMINI-300 MHz instrument, using $\mathrm{CDCl}_{3}$ as a solvent and TMS as internal reference. The IR spectra were recorded on a Perkin Elmer 1600-FTIR (film in $\mathrm{NaCl}$ cell) instrument. Elemental analyses were performed on a Perkin Elmer 2400 instrument. The mass spectra were recorded on a HP $5988^{\mathrm{A}}$ instrument. The gas chromatographic analysis were performed on a Shimadzu GC/MS Class 5000 chromatograph equipped with a Simplicity-1 (SUPELCO) column. The products were purified by flash chromatography or PLC using $\mathrm{SiO}_{2}$ as a stationary phase.

General procedure to obtain $\gamma$-amino alcohol, (1). To a solution of $\beta$-enamino ketone $(2,1 \mathrm{mmol})$ in glacial acetic acid (6 mL), was slowly added $\mathrm{NaBH}_{4}$ (4 mmol). The reaction was kept at $18-20{ }^{\circ} \mathrm{C}$. The reaction was stirred for 3 hours, and then neutralized with an aqueous solution of 
$30 \% \mathrm{NaOH}$ (approximatelly $12 \mathrm{~mL}$ ) in an ice bath. The reaction mixture was extracted with $\mathrm{CH}_{2} \mathrm{Cl}_{2}$, the organic phases were combined, dried over $\mathrm{MgSO}_{4}$, and concentrated.

4-(N-Phenylamino)-pentan-2-ol, (1a). IR (neat) $v_{\max } / \mathrm{cm}^{-1}$ : 3350, 3050, 3025, 2970, 2925, 1600, 1500, 1320, 1250, 1130, 750, 690. MS m/z (\%): 179(37), 164(15), 120(100), 104(7.6), 93(24), 77(22), 45(24). ${ }^{1} \mathrm{H}$ NMR syn-1a $\delta: 1.14(\mathrm{~d}$, $J 6.2 \mathrm{~Hz}, 3 \mathrm{H}), 1.18(\mathrm{~d}, J 6.2 \mathrm{~Hz}, 3 \mathrm{H}), 1.57\left(\mathrm{AA}^{\prime} \mathrm{XY}, 2 \mathrm{H}\right)$, 3.41(s, large, $2 \mathrm{H}$ ), 3.66(sext, $J 6.5 \mathrm{~Hz}, 1 \mathrm{H}), 4.01$ (sext, $J 6.0$ $\mathrm{Hz}, 1 \mathrm{H}), 6.57-6.79(\mathrm{~m}, 3 \mathrm{H}), 7.12-7.24(\mathrm{~m}, 2 \mathrm{H}) .{ }^{13} \mathrm{C}$ NMR syn1a $\delta: 147.13,129.46,118.98,115.29,67.97,49.89,45.76$, 23.98, 21.46. ${ }^{1} \mathrm{H}$ NMR anti-1a $\delta: 1.17(2 \mathrm{~d}, 6 \mathrm{H}), 1.43-$ $1.70(\mathrm{~m}, 2 \mathrm{H}), 3.20(\mathrm{~s}$, large, $2 \mathrm{H}), 3.76(\mathrm{~m}, 1 \mathrm{H}), 3.91(\mathrm{~m}, 1 \mathrm{H})$, 6.49-6.67(m, 3H), 7.00-7.10(m, 2H). ${ }^{13} \mathrm{C}$ NMR anti-1a $\delta$ : 147.73, 129.37, 117.61, 113.73, 67.25, 46.05, 45.70, 24.04, 21.32. Anal. Calc. for $\mathrm{C}_{11} \mathrm{H}_{17} \mathrm{NO}: \mathrm{C}, 73.70 ; \mathrm{H}, 9.56 ; \mathrm{N}$, 7.82\%. Found: C, 73.91; H, 9.56; N, 7.62\%.

4-(N-Benzylamino)-pentan-2-ol, (1b). IR (neat) $v_{\max } / \mathrm{cm}^{-1}$ : 3346, 3286, 3076, 2964, 2925, 1452, 1374, 1165, 1129, 1088, 742, 698. MS m/z (\%): 193(5), 178(15), 134(83), 106(28), 91(100). ${ }^{1} \mathrm{H}$ NMR syn-1b $\delta: 1.12(\mathrm{~d}, J 6.3 \mathrm{~Hz}, 3 \mathrm{H})$, $1.17(\mathrm{~d}, J 8.1 \mathrm{~Hz}, 3 \mathrm{H}), 1.20-1.55(\mathrm{~m}, 2 \mathrm{H}), 2.92(\mathrm{dqd}, J 2.7$, $6.3,10.6 \mathrm{~Hz}, 1 \mathrm{H}), 3.71(\mathrm{~d}, J 13.0 \mathrm{~Hz}, 1 \mathrm{H}), 3.90(\mathrm{~s}$, large, $1 \mathrm{H})$, 3.93(d, $J 13.0 \mathrm{~Hz}, 1 \mathrm{H}), 3.94(\mathrm{dqd}, J 2.3,5.8,17.0 \mathrm{~Hz}, 1 \mathrm{H})$, 7.20-7.36(m, 5H). ${ }^{13} \mathrm{C}$ NMR syn-1b $\delta: 139.24,128.72$, 128.49, 127.48, 68.94, 54.08, 50.64, 44.91, 23.87, 20.82. ${ }^{1} \mathrm{H}$ NMR anti-1b $\delta: 1.15(\mathrm{~d}, J 8.1 \mathrm{~Hz}, 3 \mathrm{H}), 1.21(\mathrm{~d}, J 6.6 \mathrm{~Hz}$, $3 \mathrm{H}$ ), 1.43(ddd, J 2.7, 5.1, $14.4 \mathrm{~Hz}, 1 \mathrm{H}$ ), 1.70(ddd, J 3.3, 9.0, $14.4 \mathrm{~Hz}, 1 \mathrm{H}), 3.12(\mathrm{dqd}, J 3.2,3.9,6.6 \mathrm{~Hz}, 1 \mathrm{H}), 3.50(\mathrm{~s}$, large, $2 \mathrm{H}), 3.74(\mathrm{~d}, J 12.6 \mathrm{~Hz}, 1 \mathrm{H}), 3.86(\mathrm{~d}, J 12.6 \mathrm{~Hz}, 1 \mathrm{H})$, 4.15(dqd, J 3.0, 6.2, 9.0 Hz, 1H), 7.20-7.37(m, 5H). ${ }^{13} \mathrm{C}$ NMR anti-1b $\delta: 139.51,128.73,128.43,127.45,65.03$, 51.48, 51.97, 45.06, 23.56, 19.76. Anal. Calc. for $\mathrm{C}_{12} \mathrm{H}_{19} \mathrm{NO}$ : C, 74.55; H, 9.91; N, 7.25\%. Found: C, 74.48; H, 10.17; N, $7.31 \%$.

4-(N-isopropylamino)-pentan-2-ol, (1c). IR (neat) $v_{\max } / \mathrm{cm}^{-1}$ : 3340, 3274, 2965, 2927, 1560, 1461, 1382, 1163, 1133, 1084. MS $m / z$ (\%): 145(0.2), 130(15), 86(65), 45(25). ${ }^{1} \mathrm{H}$ NMR syn-1c $\delta: 1.08(\mathrm{~d}, J 6.0 \mathrm{~Hz}, 3 \mathrm{H}), 1.10(\mathrm{~d}, J 5.6 \mathrm{~Hz}, 3 \mathrm{H})$, $1.11(\mathrm{~d}, J 6.2 \mathrm{~Hz}, 3 \mathrm{H}), 1.14(\mathrm{~d}, J 6.2 \mathrm{~Hz}, 3 \mathrm{H}), 1.20-1.40(\mathrm{~m}$, $1 \mathrm{H}), 1.53$ (ddd, J 2.2, 2.8, $14.0 \mathrm{~Hz}, 1 \mathrm{H}$ ), 3.00(hept, $J 6.4 \mathrm{~Hz}$, $1 \mathrm{H}), 3.05(\mathrm{~m}, 1 \mathrm{H}), 3.97(\mathrm{dqd}, J 1.8,6.2,10.7 \mathrm{~Hz}, 1 \mathrm{H}), 4.30(\mathrm{~s}$, large, 2H). ${ }^{13} \mathrm{C}$ NMR syn-1c $\delta: 69.07,51.68,45.35,45.03$, 24.23, 24.01, 21.72, 21.13. ${ }^{1} \mathrm{H}$ NMR anti-1c $\delta: 1.10(\mathrm{~d}, J$ $6.2 \mathrm{~Hz}, 3 \mathrm{H}), 1.13(\mathrm{~d}, J 6.3 \mathrm{~Hz}, 3 \mathrm{H}), 1.18(\mathrm{~d}, J 6.3 \mathrm{~Hz}, 3 \mathrm{H})$, $1.20(\mathrm{~d}, J 6.6 \mathrm{~Hz}, 3 \mathrm{H}), 1.20-1.40(\mathrm{~m}, 1 \mathrm{H}), 1.65(\mathrm{ddd}, J 3.3$, 8.1, $13.0 \mathrm{~Hz}, 1 \mathrm{H}), 3.00$ (hept, $J 6.4 \mathrm{~Hz}, 1 \mathrm{H}), 3.26(\mathrm{dqd}, J 3.8$, 6.3, 6.7 Hz, 1H), 4.16(dqd, J 3.2, 5.9, 8.4 Hz, 1H), 4.30(s, large, $2 \mathrm{H}) .{ }^{13} \mathrm{C}$ NMR anti-1c $\delta: 65.03,48.25,45.77,41.23$, 23.51, 22.70, 22.05, 19.51 .
1-Phenyl-3-(N-isopropylamino)-butan-1-ol, (1d). IR (neat) $v_{\max } / \mathrm{cm}^{-1}: 3270,3200,3040,3020,2980,2880$, 1470, 1380, 1330, 1100, 1070, 750, 700. MS m/z (\%): 207(4.6), 192(2), 107(4.6), 105(6.9), 86(100), 77(20), 70(37). ${ }^{1} \mathrm{H}$ NMR syn-1d $\delta: 1.30-1.35(3 \mathrm{~d}, 9 \mathrm{H}), 1.67(\mathrm{td}, J$ $10.7,14.5 \mathrm{~Hz}, 1 \mathrm{H}), 1.92(\mathrm{td}, J 2.5,14.5 \mathrm{~Hz}, 1 \mathrm{H}), 3.21$ (hept, $J 6.2 \mathrm{~Hz}, 1 \mathrm{H}), 3.18-3.35(\mathrm{dqd}, J 2.6,5.8,7.0 \mathrm{~Hz}, 1 \mathrm{H}), 4.47(\mathrm{~s}$, large, $2 \mathrm{H}), 5.07(\mathrm{dd}, J 1.5,10.6 \mathrm{~Hz}, 1 \mathrm{H}), 7.37-7.60(\mathrm{~m}, 5 \mathrm{H})$. ${ }^{13}$ C NMR syn-1d $\delta: 145.55,128.11,126.79,125.53,75.08$, 51.63, 46.38, 45.30, 24.39, 21.93, 21.83. ${ }^{1} \mathrm{H}$ NMR anti-1d $\delta: 1.00(\mathrm{~d}, J 6.3 \mathrm{~Hz}, 3 \mathrm{H}), 1.03(\mathrm{~d}, J 6.3 \mathrm{~Hz}, 3 \mathrm{H}), 1.07(\mathrm{~d}, J 6.3$ $\mathrm{Hz}, 3 \mathrm{H})$, 1.65(ABMX, J3.9, 7.0, 14.0 Hz, 1H), 1.74(ABMX, $J$ 3.6, 6.0, $15.0 \mathrm{~Hz}, 1 \mathrm{H}), 2.82$ (hept, J $6.3 \mathrm{~Hz}, 1 \mathrm{H}), 2.90(\mathrm{~m}$, 1H), 3.82(s, large, 2H), 4.88(dd, J 3.7, $6.7 \mathrm{~Hz}, 1 \mathrm{H}), 7.06-$ 7.30(m, 5H). ${ }^{13} \mathrm{C}$ NMR anti-1d $\delta: 150.09,131.91,130.34$, 129.71, 75.19, 51.93, 49.63, 47.58, 27.83, 26.82, 24.70. Anal. Calc. for $\mathrm{C}_{13} \mathrm{H}_{21} \mathrm{NO}$ : C, 75.32; $\mathrm{H}, 10.21 ; \mathrm{N}, 6.75 \%$. Found: C, 75.10; H, 10.22; N, 6.26\%.

4-(N-Pyrrolidinyl)-pentan-2-ol, (1e). IR (neat) $v_{\max } / \mathrm{cm}^{-1}$ : 3400, 2980, 2940, 2880, 2830, 1455, 1160, 1150. MS $\mathrm{m} / \mathrm{z}(\%):$ 157(4.2), 142(9), 96(100), 70(24), 56(20), 45(24). ${ }^{1} \mathrm{H}$ NMR syn-1e $\delta$ : $0.97(\mathrm{~d}, J 6.6 \mathrm{~Hz}, 3 \mathrm{H}), 1.13(\mathrm{~d}, J 6.2 \mathrm{~Hz}$, $3 \mathrm{H}), 1.34(\mathrm{ddd}, J 1.8,3.6,14.5 \mathrm{~Hz}, 1 \mathrm{H}), 1.52(\mathrm{td}, J 10.9$, $14.0 \mathrm{~Hz}, 1 \mathrm{H}), 1.70-1.80\left(\mathrm{AA}^{\prime} \mathrm{B}_{2}, 4 \mathrm{H}\right), 2.57-2.80\left(\mathrm{AA}^{\prime} \mathrm{B}_{2}, 4 \mathrm{H}\right)$, 3.17(dqd, $J 3.3,6.9,10.5 \mathrm{~Hz}, 1 \mathrm{H}), 3.96(\mathrm{dqd}, J$ 2.0, 6.2, $10.3 \mathrm{~Hz}, 1 \mathrm{H}), 6.24(\mathrm{~s}$, large, $1 \mathrm{H}) .{ }^{13} \mathrm{C}$ NMR syn-1e $\delta: 69.08$, 55.56, 46.70, 41.94, 23.67, 23.23, 12.43. ${ }^{1} \mathrm{H}$ NMR anti-1e $\delta: 1.21(\mathrm{~d}, J 6.2 \mathrm{~Hz}, 3 \mathrm{H}), 1.25(\mathrm{~d}, J 6.6 \mathrm{~Hz}, 3 \mathrm{H}), 1.55(\mathrm{ddd}, J$ 2.6, 7.0, $14.0 \mathrm{~Hz}, 1 \mathrm{H}), 1.83(\mathrm{ddd}, J 6.1,10.6,14.0 \mathrm{~Hz}, 1 \mathrm{H})$, 1.94-2.20(m, 4H), 3.09(t, J 6.5 Hz, 4H), 3.50(sext, J $6.6 \mathrm{~Hz}$, $1 \mathrm{H}), 3.90(\mathrm{dqd}, J 1.8,5.6,11.0 \mathrm{~Hz}, 1 \mathrm{H}), 5.20(\mathrm{~s}$, large, $1 \mathrm{H})$. ${ }^{13} \mathrm{C}$ NMR anti-1e $\delta: 64.43,57.62,51.48,41.05,23.52$, 23.28, 17.28.

General procedure to obtain tetrahydro-1,3-oxazines, (3). To a solution of $\gamma$-amino alcohol $(\mathbf{1}, 1 \mathrm{mmol})$ in diethyl ether $(1 \mathrm{~mL})$, was added a solution of $40 \%$ formaldehyde $(0.1 \mathrm{~mL})$. The reaction was stirred for 16-20 hours at room temperature. After this time, diethyl ether (approximatelly $5 \mathrm{~mL}$ ) was added, and the solution was dried over $\mathrm{MgSO}_{4}$, filtered and concentrated in vacuo. The yield was quantitative.

3-Phenyl-4,6-dimethyl-tetrahydro-1,3-oxazine, (3a). IR (neat) $v_{\max } / \mathrm{cm}^{-1}: 2960,2920,1600,1485,1370,1250$, 1240, 1175, 1100, 1000, 700. MS m/z (\%): 192(7), 191(50), 190(11), 176(58), 132(83), 120(50), 119(83), 118(22), 106(33), 105(83), 104(91), 91(14), 77(100). ${ }^{1} \mathrm{H}$ NMR syn3a $\delta: 1.02(\mathrm{~d}, J 6.3 \mathrm{~Hz}, 3 \mathrm{H}), 1.26(\mathrm{~d}, J 6.3 \mathrm{~Hz}, 3 \mathrm{H}), 1.42(\mathrm{dt}$, $J 11.1,13.0 \mathrm{~Hz}, 1 \mathrm{H}), 1.57(\mathrm{dt}, J 2.7,13.5 \mathrm{~Hz}, 1 \mathrm{H}), 3.73(\mathrm{ddq}$, $J 3.3,6.3,11.1 \mathrm{~Hz}, 1 \mathrm{H}), 3.68(\mathrm{ddq}, J 2.7,6.3,12.3 \mathrm{~Hz}, 1 \mathrm{H})$, 4.39(d, J 9.3 Hz, 1H), 4.73(d, J 9.3 Hz, 1H), 7.06-7.33(m, $5 \mathrm{H}) .{ }^{13} \mathrm{C}$ NMR syn-3a $\delta: 147.57,129.02,126.32,124.83$, 
85.76, 73.43, 53.90, 40.03, 21.70, 20.04. ${ }^{1} \mathrm{H}$ NMR anti-3a $\delta: 1.16(\mathrm{~d}, J 6.3 \mathrm{~Hz}, 3 \mathrm{H}), 1.25(\mathrm{dt}, J 2.0,13.0 \mathrm{~Hz}, 1 \mathrm{H}), 1.41(\mathrm{~d}$, $J 6.9 \mathrm{~Hz}, 3 \mathrm{H}), 1.75(\mathrm{ddd}, J 5.4,12.0,13.8 \mathrm{~Hz}, 1 \mathrm{H}), 3.95(\mathrm{~m}$, $2 \mathrm{H}), 4.83(\mathrm{~d}, J 11.1 \mathrm{~Hz}, 1 \mathrm{H}), 4.98(\mathrm{~d}, J 11.4 \mathrm{~Hz}, 1 \mathrm{H}), 6.85(\mathrm{t}$, $J 8.4 \mathrm{~Hz}, 1 \mathrm{H}), 7.03(\mathrm{~d}, J 7.8 \mathrm{~Hz}, 2 \mathrm{H}), 7.22(\mathrm{dd}, J 7.2,8.7 \mathrm{~Hz}$, 2H). ${ }^{13} \mathrm{C}$ NMR anti-3a $\delta: 150.88,129.35,120.65,119.08$, 74.85, 68.14, 52.79, 35.76, 22.07, 17.07. Anal. Calc. for $\mathrm{C}_{12} \mathrm{H}_{17} \mathrm{NO}$ : C, 75.35; H, 8.96; N, 7.32\%. Found: C, 74.80; $\mathrm{H}, 8.80 ; \mathrm{N}, 7.37 \%$.

3-Benzyl-4,6-dimethyl-tetrahydro-1,3-oxazine, (3b). IR (neat) $v_{\max } / \mathrm{cm}^{-1}: 2980,2940,1450,1370,1200,1050,740$, 700. MS m/z (\%): 206(3), 205(17), 204(8), 190(38), 146(14), 92(42), 91(100). ${ }^{1} \mathrm{H}$ NMR syn-3b $\delta: 1.13(\mathrm{~d}, J 6.0 \mathrm{~Hz}, 6 \mathrm{H})$, $1.20-1.40(\mathrm{~m}, 2 \mathrm{H}), 2.95(\mathrm{~m}, 1 \mathrm{H}), 3.47(\mathrm{~d}, J 13.5 \mathrm{~Hz}, 1 \mathrm{H}), 3.47(\mathrm{~m}$, $1 \mathrm{H}), 3.79(\mathrm{~d}, J 13.5 \mathrm{~Hz}, 1 \mathrm{H}), 3.92(\mathrm{~d}, J 10.2 \mathrm{~Hz}, 1 \mathrm{H}), 4.21(\mathrm{~d}, J$ $10.2 \mathrm{~Hz}, 1 \mathrm{H}), 7.20-7.40$ (AA'BBC, 5H). ${ }^{13} \mathrm{C}$ NMR syn-3b $\delta$ : 139.43, 128.39, 127.74, 126.32, 83.00, 72.79, 55.29, 48.92, 37.29, 21.74, 20.16. ${ }^{1} \mathrm{H}$ NMR anti-3b $\delta: 1.17(\mathrm{~d}, J 6.0 \mathrm{~Hz}$, $3 \mathrm{H}), 1.25(\mathrm{~d}, J 6.0 \mathrm{~Hz}, 3 \mathrm{H}), 1.10-1.20(\mathrm{AXYZ}, 1 \mathrm{H}), 1.88(\mathrm{AXYZ}$, 1H), 2.98(q, J $6.0 \mathrm{~Hz}, 1 \mathrm{H}), 3.81(\mathrm{dqd}, 1 \mathrm{H}), 3.96\left(\mathrm{AA}^{\prime}, 2 \mathrm{H}\right)$, $4.25(\mathrm{~d}, J 11.0 \mathrm{~Hz}, 1 \mathrm{H}), 4.65(\mathrm{~d}, J 11.0 \mathrm{~Hz}, 1 \mathrm{H}), 7.40-7.20(\mathrm{~m}$, 5H). ${ }^{13} \mathrm{C}$ NMR anti-3b $\delta: 139.67,128.50,128.23,126.89$, 78.47, 67.80, 56.92, 49.28, 32.58, 22.20, 18.10. Anal. Calc. for $\mathrm{C}_{13} \mathrm{H}_{19} \mathrm{NO}$ : C, 76.06; H, 9.33; N, 6.82\%. Found: C, 76.58; $\mathrm{H}, 9.23 ; \mathrm{N}, 6.60 \%$.

3-Isopropyl-4,6-dimethyl-tetrahydro-1,3-oxazine, (3c). MS m/z (\%): 157(20), 142(100), 114(9), 100(14), 98(45), 56(81). ${ }^{1} \mathrm{H}$ NMR syn-3c $\delta: 0.92(\mathrm{~d}, J 6.6 \mathrm{~Hz}, 3 \mathrm{H}), 1.09(\mathrm{~d}, J$ $6.6 \mathrm{~Hz}, 6 \mathrm{H}), 1.15(\mathrm{~d}, J 6.9 \mathrm{~Hz}, 3 \mathrm{H}), 1.03-1.24(\mathrm{~m}, 2 \mathrm{H})$, $2.75(\mathrm{dqd}, J 3.0,12.0 \mathrm{~Hz}, 1 \mathrm{H}), 3.22$ (hept, $J 6.0 \mathrm{~Hz}, 1 \mathrm{H}$ ), 3.39 (dqd, $J 3.0,6.0,12.0 \mathrm{~Hz}, 1 \mathrm{H}), 3.82(\mathrm{~d}, J 8.7 \mathrm{~Hz}, 1 \mathrm{H})$, 4.49(d, J 9.0 Hz, 1H). ${ }^{13} \mathrm{C}$ NMR syn-3c $\delta: 78.47,72.65$, 52.55, 45.03, 41.38, 21.98, 21.68, 19.63, 17.18. ${ }^{1} \mathrm{H}$ NMR anti-3c $\delta: 1.02-1.24(\mathrm{~m}, 1 \mathrm{H}), 1.09(\mathrm{~d}, J 6.6 \mathrm{~Hz}, 6 \mathrm{H}), 1.27(\mathrm{~d}$, $J 6.3 \mathrm{~Hz}, 3 \mathrm{H}), 1.99(\mathrm{~d}, J 7.2 \mathrm{~Hz}, 3 \mathrm{H}), 1.62(\mathrm{~m}, 1 \mathrm{H}), 3.03$ (hept, $J 6.0 \mathrm{~Hz}, 1 \mathrm{H}$ ), 3.15(quint, $J 6.0 \mathrm{~Hz}, 1 \mathrm{H}$ ), 3.65(dqd, $J 3.0$, 6.0, $10.5 \mathrm{~Hz}, 1 \mathrm{H}), 4.36\left(\mathrm{AA}^{\prime}, 2 \mathrm{H}\right) .{ }^{13} \mathrm{C} \mathrm{NMR}$ anti-3c $\delta: 75.72$, 67.20, 51.07, 46.92, 34.72, 22.81, 22.33, 21.82, 18.87.

6-Phenyl-3-isopropyl-4-methyl-tetrahydro-1,3-oxazine, (3d). IR (neat) $v_{\max } / \mathrm{cm}^{-1}: 2967,2922,2871,1604,1495$, 1452, 1383, 1210, 1079. MS $m / z(\%): 220(4.3), 219(26.5)$, 205(10.6), 204(67.4), 174(5.6), 140(5.2). ${ }^{1} \mathrm{H}$ NMR syn-3d $\delta: 1.02(\mathrm{~d}, J 6.4 \mathrm{~Hz}, 3 \mathrm{H}), 1.20(\mathrm{~d}, J 6.5 \mathrm{~Hz}, 3 \mathrm{H}), 1.26(\mathrm{~d}, J 6.7$ Hz, 3H), 1.69(AA'Y, 2H), 2.99(dqd, J3.2, 6.4, 10.7 Hz, 1H), 3.36(hept, $J 6.6 \mathrm{~Hz}, 1 \mathrm{H}), 4.45(\mathrm{dd}, J 3.7,10.6 \mathrm{~Hz}, 1 \mathrm{H}), 4.65(\mathrm{~d}$, $J 8.9 \mathrm{~Hz}, 1 \mathrm{H}), 4.84(\mathrm{~d}, J 8.9 \mathrm{~Hz}, 1 \mathrm{H}), 7.21-7.45(\mathrm{~m}, 5 \mathrm{H}) .{ }^{13} \mathrm{C}$ NMR syn-3d $\delta: 142.63,128.42,127.51,125.96,79.76$, 79.68, 53.13, 45.67, 41.89, 21.96, 19.72, 16.93. ${ }^{1} \mathrm{H}$ NMR anti-3d $\delta: 1.14(\mathrm{~d}, J 6.4 \mathrm{~Hz}, 3 \mathrm{H}), 1.18(\mathrm{~d}, J 6.5 \mathrm{~Hz}, 3 \mathrm{H})$, 1.34(d, J 7.2 Hz, 3H), 1.60(m, 1H), 1.93(ddd, J 6.0, 11.8, $13.4 \mathrm{~Hz}, 1 \mathrm{H}$ ), 3.15 (quint, J $6.6 \mathrm{~Hz}, 1 \mathrm{H}$ ), $3.25(\mathrm{~m}, J 6.3 \mathrm{~Hz}$,
1H), 4.59(AA', 2H), 4.60(dd, J 3.2, 9.7 Hz, 1H), 7.20(m, 5H). ${ }^{13} \mathrm{C}$ NMR anti-3d $\delta: 143.57,127.91,126.78,125.38$, 76.33, 73.57, 51.68, 47.31, 35.55, 22.86, 21.95, 18.80 . Anal. Calc. for $\mathrm{C}_{14} \mathrm{H}_{21} \mathrm{NO}$ : C, 76.06; H, 9.33; N, 6.82\%. Found: C, 76.58; H, 9.23; N, 6.60\%.

3-Benzyl-4-methyl-6-terc-butyl-tetrahydro-1,3-oxazine, (3f). IR (neat) $v_{\max } / \mathrm{cm}^{-1}: 2956,2869,1188,1105,1027$, 734, 698. MS m/z (\%): 232(9), 190(12), 146(15), 118(4), 91(100). ${ }^{1} \mathrm{H}$ NMR syn-3f $\delta: 0.71(\mathrm{~s}, 9 \mathrm{H}), 1.02(\mathrm{~d}, J 6.6 \mathrm{~Hz}$, $3 \mathrm{H}), 1.12(\mathrm{dt}, J 2.8,11.3 \mathrm{~Hz}, 1 \mathrm{H}), 1.29$ (AXYZ, 1H), 2.79(dqd, $J 3.0,6.6,11.3 \mathrm{~Hz}, 1 \mathrm{H}), 2.87(\mathrm{dd}, J 11.3,2.6 \mathrm{~Hz}, 1 \mathrm{H}), 3.35(\mathrm{~d}$, $J 13.5 \mathrm{~Hz}, 1 \mathrm{H}), 3.65(\mathrm{~d}, J 13.5 \mathrm{~Hz}, 1 \mathrm{H}), 3.83(\mathrm{dd}, J 0.9,9.8$ $\mathrm{Hz}, 1 \mathrm{H}), 4.20(\mathrm{~d}, J 9.8 \mathrm{~Hz}, 1 \mathrm{H}), 6.93-7.21(\mathrm{~m}, 5 \mathrm{H}) .{ }^{13} \mathrm{C} \mathrm{NMR}$ syn-3f $\delta: 139.34,128.74,127.95,126.56,84.55,83.34$, $55.07,48.26,34.01,29.41,25.64,20.44$.

\section{Acknowledgment}

The authors thank the FINEP-Financiadora de Estudos e Projetos for financial support.

\section{References}

1. For a general review see: Gevorgyan, G. A.; Agababian, G.; Russ. Chem. Rev. 1984, 53, 581; Jäger, V.; Buß, V.; Liebigs Ann. Chem. 1980, 101; Wang, Y. F.; Izawa, T.; Kobayashi, S.; Ohno, M.; J. Am. Chem. Soc. 1982, 104, 6465; Heitsch, H.; König, W. A.; Decker, H.; Bormann, C.; Fiedler, H. P.; Zähner, H.; J. Antibiotics 1989, 42, 711; Wovkulich, P. M.; Uskokovic, M. R.; J. Am. Chem. Soc. 1981, 103, 3956; Jäger, V.; Schwab, W.; Bub, V.; Angew. Chem. Int. Ed. Engl. 1981, 20, 601.

2. White, D. W.; Gibbs, D. E.; Verkade, J. G.; J. Am. Chem. Soc. 1979, 101, 1937.

3. Liguori, A.; Romeo, G.; Sindona, G.; Ucella, N.; Chem. Ber. 1988, 121, 105.

4. Bongini, A.; Cardillo, G.; Orena, M.; Porzi, G.; Sandri, S.; Chem. Lett. 1988, 87.

5. Arisa, J.; Font, J.; Ortuño, R. M.; Tetrahedron 1990, 46, 1931.

6. Narasaka, K.; Ukaji, Y.; Yamazaki, S.; Bull. Chem. Soc. Jpn. 1986, 59, 525 .

7. Maroni, P.; Cazaux, L.; Tisnes, P.; Zambeti, M.; Bull. Soc. Chim. Fr. P II 1980, 179.

8. Matsumura, Y.; Fujiwara, J.; Maruoka, K.; Yamamoto, H.; J. Am. Chem. Soc. 1983, 105, 6312.

9. Bartoli, G.; Cimarelli, C.; Palmieri, G.; J. Chem. Soc., Perkin Trans. 1 1994, 537.; Bartoli, G.; Cupone, G.; Dalpozzo, R.; De Nino, A.; Maiuolo, L.; Procopio, A.; Tagarelli, A.; Tetrahedron Lett. 2002, 43, 7441.

10. Greenhill, J. V.; Chem. Soc. Rev. 1977, 6, 277.

11. Schuda, P. F.; Ebner, C. B.; Morgan, T. M.; Tetrahedron Lett. 1986, 27, 2567. 
12. Barluenga, J.; Aguilar, E.; Fustero, S.; Olano, B.; Viado, A. L.; J. Org. Chem. 1992, 57, 1219.

13. Katritzky, A.; Harris, P. A.; Tetrahedron 1990, 46, 987.

14. Pilli, R. A.; Russowsky, D.; Dias, L. C.; J. Chem. Soc., Perkin Trans. 1 1990, 1213.

15. Tramontini, M.; Synthesis 1982, 605.

16. For a comprehensive review of uses of borohydrides in carboxylic acid media see: Gribble, G. W.; Nutaitis, C. F.; Org. Prep. Proc. Int. 1985, 17, 317; Gribble, G. W.; Chem. Soc. Rev. 1998, 27, 395.

17. Harris, M. I. N. C.; PhD. Thesis, Universidade Estadual de Campinas, Brazil, 1993; Braga, A. C. H.; Harris, M. I. N. C.; Br PI 9.502.467-0, 1995. (CA 128:243740).
18. It is well known that the reaction of $\mathrm{NaBH}_{4}$ with neat carboxilic acids or solutions of carboxilic acids in nonprotic solvents leads to the formation of acyloxyborohydrides. ${ }^{16}$ In order to understand the real nature of the reducing agent, studies with sodium triacetoxyborohydride are in progress.

19. Barluenga, J.; Olano, B.; Fustero, S.; J. Org. Chem. 1985, 50, 4052.

Received: March 9, 2004

Published on the web: September 28, 2004

FAPESP helped in meeting the publication costs of this article. 